\title{
Ataque de Coptoborus ochromactonus (Coleoptera: Curculionidae: Scolytinae) en plantaciones de Ochroma pyramidale (Cav. ex Lam.)
}

\section{Access of Coptoborus ochromactonus (Coleoptera: Curculionidae: Scolytinae) in plantations the Ochroma pyramidale (Cav. ex Lam.)}

\author{
${ }^{\bullet}$ Jessenia Castro Olaya ${ }^{1,2}$, Dolly Lanfranco Leverton ${ }^{2}$, Malena Martinez Chévez ${ }^{1}$, \\ Marcelino Guachambala ${ }^{3}$, Carlos Belezaca Pinargote ${ }^{1}$, Anna A. Obrebska ${ }^{4}$ \\ ${ }^{1}$ Universidad Técnica Estatal de Quevedo. Facultad de Ciencias Ambientales. Carrera de Ingeniería Forestal. Campus Ing. \\ Manuel Haz Álvarez, km 1.5 vía a Santo Domingo de los Tsáchilas. EC.120501. Quevedo, Ecuador. \\ ○jcastroo@uteq.edu.ec; mmartinez@uteq.edu.ec; cbelezaca@uteq.edu.ec \\ ${ }^{2}$ Universidad Austral de Chile, Facultad de Ciencias Forestales y Recursos Naturales. Campus Isla Teja, Casilla 567. \\ Valdivia, Chile.dlanfran@uach.cl \\ ${ }^{3}$ Plantaciones de Balsa Plantabal S. A. 3AComposites. Km 5 via Valencia. Valencia, Ecuador. marcelino36_1@hotmail.com \\ ${ }^{4}$ Instituto Nacional de Investigaciones Agropecuarias-INIAP. Estación Experimental Tropical Pichilingue. CP. 121250. \\ Km 5 1/2 vía Quevedo-El Empalme, cantón Mocache, Los Ríos. Ecuador. anna.obrebska31@gmail.com
}

Rec.: 03.04.2017. Acept.: 25.08.2017. Publicado el 1 de diciembre de 2017

\section{Resumen}

C Toptoborus ochromactonus, es un escolítido asociado al cultivo de balsa (Ochroma pyramidale), que causa perforaciones y construye galerías en fuste y ramas. La incidencia de daño puede llegar al $50 \%$ en una plantación y de estos causa un $15 \%$ de mortalidad. Los objetivos del presente trabajo fueron caracterizar el daño, definir la incidencia en plantaciones de 24 meses de edad en las localidades de Buena Fe y El Empalme y evaluar las características dasométricas del árbol hospedero. Se seleccionaron 12 parcelas circulares en cada plantación, donde previo a observaciones se realizó una categorización, árboles sin daño, daño 1 y daño 2. En cada parcela se evaluaron todos los árboles, registrando las diferentes categorías y las variables DAP (diámetro a la altura del pecho) y altura total. La incidencia se evaluó mediante la comparación del total de árboles con los de las distintas categorías. El daño 1 es dirigido al fuste, donde se observan perforaciones con exudación. En la plantación de Buena Fe se obtuvo el $9.80 \%$ y en El Empalme fue de $22.60 \%$. Mientras que el daño 2, que es dirigido a las ramas de la copa y en ellos se observa la presencia de aserrín y marchitez del follaje, se observó solo en Buena Fe con 0.80\%. La incidencia de daño fue mayor en El Empalme con el 22.60\%, atacando árboles de $20.10 \mathrm{~cm}$ de DAP y $18.40 \mathrm{~m}$ de alto, en relación a Buena Fe, con el $10.64 \%$, atacando árboles de $18.20 \mathrm{~cm}$ de DAP y 15.90 $\mathrm{m}$ de alto.

Palabras clave: Escolítido, balsa, muerte regresiva, incidencia, características dasométricas.

\begin{abstract}
Coptoborus ochromactonus, is a recently described scolytid $\checkmark$ pest associated with the balsa plantations, causing damage and death in young trees (from 12 to 36 months). The objectives were: Characterize the distribution of damage into the tree, define the incidence of $C$. ochromactonus in two years old plantations in Buena Fe and El Empalme, and evaluate dasometrics parameters of its host. C. ochromactonus has two types of damages that correspond to the initial attack, when the shaft is unsuccessful and no mass attack, whereas when it occurs in upper branches and exceed 300 drillings produces symptoms of dieback and then spike the shaft. The incidence of injury was higher in El Empalme 22.90\%, attacking trees $20.10 \mathrm{~cm} \mathrm{dbh}$ and $18.40 \mathrm{~m}$ high, relative to Buena Fe, with $11.20 \%, 18.20$ attacking trees $\mathrm{dbh} \mathrm{cm}$ and $15.90 \mathrm{~m}$ high
\end{abstract}

Key words: Scolytido, balsa, die back, incidence, dasometric parameters. 
Introducción

C Ooptoborus ochromactonus Smith y Cognato (2014), es un insecto de ambrosía presente en plantaciones de Ochroma pyramidale (Cav. Ex. Lam.) Urb (balsa). Aunque en 1990 se registraron daños por escolítidos en plantaciones de balsa ubicadas aproximadamente a $40 \mathrm{~km}$ del noreste de Quevedo, la caracterización de daño para $C$. ochromactonus se realizó en el año 2006, caracterizados por la presencia de orificios de entrada, marchitez foliar y posteriormente la muerte regresiva del árbol. En el 2014 se describió y registró esta especie para el género Coptoborus (Stilwell et al., 2014). En este mismo año, indican que las infestaciones al parecer se inician en las ramas superiores de la balsa, y un aumento del número de ataque exitoso provoca la marchitez del follaje y muerte de la rama, si el ataque es agresivo el árbol puede llegar a la muerte. Los daños causados por C. ochromactonus pueden llegar al $31.70 \%$ de incidencia y provocar una mortalidad del 15.90\%. Ataca árboles jóvenes entre los 18 y 36 meses, y los diámetros de preferencia de ataque son entre $16.90 \mathrm{y}$ $22.10 \mathrm{~cm}$ (Stilwell et al., 2014).

El árbol hospedero, Ochroma pyramidale, presenta una distribución natural desde el sur de México hasta Brasil, y está naturalizada en África, India y Oceanía (Brink, 2008). El desarrollo industrial está centrado en Brasil, Colombia, Papúa Nueva Guinea y Ecuador, este último cubre el $95 \%$ de la demanda de la madera de balsa a nivel mundial (Fletcher, 1949; Brink, 2008; Midgley et al., 2010). En Ecuador, la distribución natural es desde 0 a $1.000 \mathrm{msnm}$, alcanzando entre los cinco y seis años de edad un diámetro aproximado de $30 \mathrm{~cm}$ y una altura entre 18-25 metros (Fletcher, 1949; Bonet et al., 2013). En los últimos años se reportan más de 20 mil hectáreas entre bosques naturales y plantaciones, concentradas principalmente en las provincias de Esmeraldas, Santo Domingo de los Tsachilas, Guayas, Los Ríos y Cotopaxi (González et al., 2010; Bonet et al., 2013).

Los daños ocasionados por el insecto ponen en riesgo la producción de madera de balsa en Ecuador, si bien existe información de los daños causados por ese insecto, un reconocimiento en detalle de su modo de ataque, así como el comportamiento en otras localidades, proporcionará información valiosa para el control y prevención de ataques en las localidades donde se cultiva la balsa. El presente trabajo se realizó para caracterizar el daño de C. ochromactonus en plantaciones, definir la incidencia de ataque en plantaciones de balsa de dos años en las localidades de El Empalme y Buena Fe, y determinar las características dasométricas de los árboles hospederos que ataca.

\author{
Materiales y métodos
}

T as mediciones y evaluaciones se realizaron en los meses $d$ del periodo lluvioso en la costa ecuatoriana (enero, febrero y marzo del 2013), en dos plantaciones de dos años de edad. Las plantaciones estuvieron ubicadas en una zona considerada de clima húmedo tropical, cantón Buena Fe, provincia Los Ríos (053'35"S; 79²9'27"O) a $103 \mathrm{msnm}$, y la otra, en una zona de clima seco tropical, cantón El Empalme, provincia del Guayas ( $\left.1^{\circ} 02^{\prime} 46^{\prime \prime} \mathrm{S} ; 79^{\circ} 38^{\prime} 01^{\prime \prime} \mathrm{O}\right)$ a $71 \mathrm{msnm}$. Cada plantación tenía un área aproximada de 70 hectáreas, y un diseño de siembra a tres bolillo de $3 \times 4$ $\mathrm{m}$, raleo del 25\% y una densidad de 611 y 670 árboles por hectárea en Buena Fe y El Empalme, respectivamente.

Se seleccionaron 12 parcelas permanentes de crecimiento (PPC) circulares de $500 \mathrm{~m}^{2}$, de las 18 PPC establecidas en las plantaciones. Se realizó una observación directa de los daños causados por C. ochromactonus, para definir una categorización de acuerdo a la metodología usada por Lee et al. (2011). Se clasificaron como aparentemente sano ( $\sin$ follaje marchito y evidencia de perforación), daño 1 (sin follaje marchito y evidencia de perforación en el fuste) y daño 2 (follaje marchito y evidencia de perforación). Los árboles con daño 2 se caracterizaron mediante el conteo de perforaciones, se seleccionaron cinco árboles que se dividieron en trozas de cuatro metros de largo y en ellos se contabilizó el número de perforaciones. Se evaluaron todos los árboles de las parcelas mediante observaciones directas, registrando los diferentes daños, también se midió el DAP con cinta diamétrica y con clinómetro Suunto la altura total.

Con la información obtenida de la caracterización de los daños asociados al ataque de C. ochromactonus, se calculó la incidencia, utilizando el número de árboles con daño para el total de árboles y multiplicado por 100 . Respecto al número de perforaciones en los árboles con daño 2, se realizó una comparación de medias del número de perforaciones por troza, utilizando el test de Tukey con un nivel de significancia de 0.05 . Se determinó la incidencia de daño de acuerdo al diámetro y altura usando una distribución de frecuencia. Todos los análisis se realizaron usando el programa estadístico Infostat.

\section{Resultados y discusión}

$\mathrm{E}$ n las dos plantaciones se registraron árboles sin daño aparente y con las dos categorías de daño definidas. La hembra de $C$. ochromactonus, realiza dos daños iniciales en las plantaciones, uno dirigido al fuste y otro a las ramas, 
este último coincide con el hábito biológico de las especies del género Coptoborus de alimentarse de ramas delgadas (Wood, 2007). Mientras, varios autores reportan para otros escolítidos, que la altura de ataque podría estar relacionado con el comportamiento de vuelo (Weber y McPherson, 1983; Reding et al., 2010).

La parte del árbol donde atacan inicialmente los $C$. ochromactonus, influye en la característica del daño, así, el Daño 1, que corresponde al ataque dirigido al fuste, se determina por orificios de entrada acompañados de exudaciones y follaje sano. Estos árboles, durante el tiempo de evaluación no sufrieron otro ataque y permanecieron vivos y aparentemente sanos, sin embargo, se genera un cambio estructural de la madera, debido al mecanismo de protección del hospedero, donde el tejido de reacción sella el orificio (Figura 1A y 1B). El daño 2, cuyo ataque inicial es dirigido a las ramas, los orificios de entrada presentan aserrín, indicador de un ataque exitoso, evidencia que la hembra adulta de $C$. ochromactonus ha construido la galería. Este daño influye en los síntomas que presenta el árbol, como follaje marchito y seco, el cual posteriormente muere, y al mes de estar en esta condición se observa un ataque masivo en todo el fuste (Figura 1C y 1D). Esto se relaciona con la teoría de atracción de los escolítidos hacia el hospedero, atracción en masa, causado a través de un ataque inicial (Rudinsky, 1962; Raffa et al., 1993; Wood, 2007).

Los árboles con daño tipo 2, tuvieron diámetro promedio de $17.20 \mathrm{~cm}$ y altura total de $17.10 \mathrm{~m}$. Hubo diferencias significativas $(\mathrm{P}<0.05$ : 0.0006$)$ del número de perforaciones entre las trozas. Las trozas cinco y cuatro (que correspondieron a las ramas superiores) presentaron $348 \pm 96.90$ y $180 \pm 40.80$ orificios de entrada exitosa, respectivamente, y entre ellas no difieren estadísticamente, pero con las trozas restantes si difirieron (Cuadro 1). La densidad de ataque en las ramas superiores y su relación con la marchitez del follaje, también lo ha reportado para la muerte regresiva del Té asociado a Xyleborus compactus (Eichhoff, 1875) y el roble koreano, atacado por Platypus koryoensis (Murayama), donde los árboles con muerte regresiva presentaban 150 y 500 perforaciones por $\mathrm{m}^{2}$, en estos casos cerca de la parte del árbol que atacan (Keneko et al., 1965; Lee et al., 2011).

Entre las plantaciones en estudio, se evaluaron 376 y 402 árboles en Buena Fe y El Empalme, respectivamente. El porcentaje de árboles sin daño aparente y follaje sano fue mayor en Buena $\mathrm{Fe}$ con un 89.36\%, mientras, en El Empalme fue menor, con el $77.40 \%$. El menor número de árboles sin daño aparente en El Empalme, se debió a una mayor cantidad de árboles atacados, posiblemente debido a la actividad de vuelo del insecto, el mismo que ocurre en el periodo lluvioso en esta localidad (Castro, 2016), precisamente en los meses en que se realizó el trabajo. Esto se refleja en la incidencia de daño, el cual fue mayor en la plantación de El Empalme, que corresponde a zona seca con un $22.60 \%$, mientras que en Buena Fe fue del 10.64\%, zona intermedia. Esta información difiere con lo registrado por Stilwell et al. (2014), quienes indican una mayor incidencia de daño en la zona intermedia y húmeda, mientras, en la zona seca los ataques fueron menores. Aunque la incidencia de daño fue mayor en El Empalme, todo corresponde a daño 1, es decir, árboles con ataque en el fuste. Mientras, en Buena Fe, se registraron los dos daños, donde el 9.84\% de los árboles evaluados presentaron daño 1 y apenas el $0.80 \%$ de los árboles tuvieron daño 2, es decir árboles con muerte regresiva. Aunque la incidencia de daño 2 fue baja, en la zona intermedia es donde mayor incidencia de
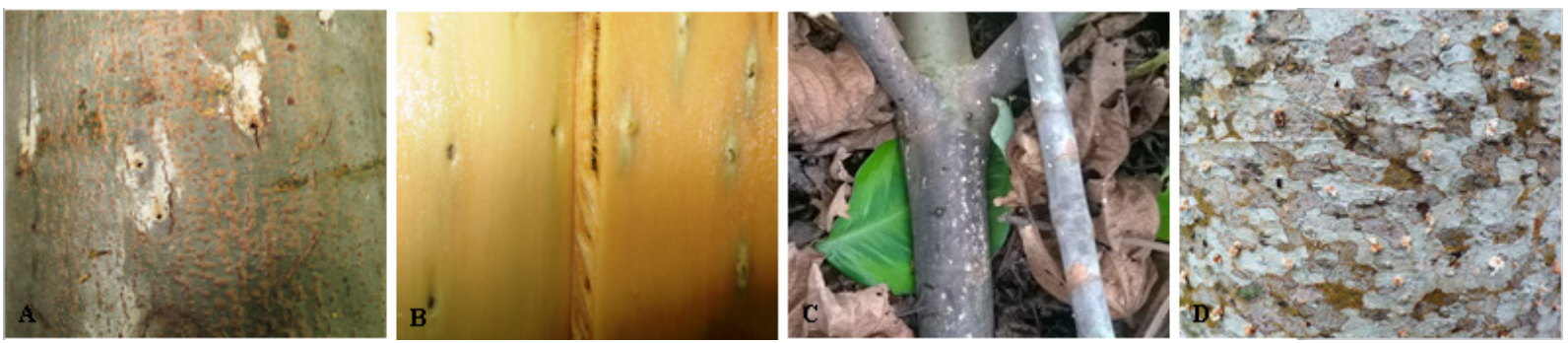

Figura 1. Daño tipo 1: A). Orificio de entrada con presencia de exudado, B). Bloqueo de entrada. Daño tipo 2: C). Rama superior con orificio de entrada y presencia de aserrín, D). ataque masivo en el fuste

Cuadro 1. Orificios de entrada por troza en árboles con daño tipo 2

\begin{tabular}{ccrcrrr}
\hline Trozas & N & largo $(\mathrm{m})$ & Media & E.E. & Mínimo & Máximo \\
\hline 1 & 5 & $1-4$ & $35 \mathrm{~b}$ & 6.40 & 19 & 56 \\
2 & 5 & $4-8$ & $42 \mathrm{~b}$ & 14.70 & 7 & 92 \\
3 & 5 & $8-13$ & $58 \mathrm{~b}$ & 17.20 & 22 & 116 \\
4 & 5 & $12-16$ & $180 \mathrm{ab}$ & 40.80 & 70 & 301 \\
5 & 5 & $16-17.50$ & $348 \mathrm{a}$ & 96.90 & 110 & 608 \\
\hline
\end{tabular}

Medias con una letra común, no son significativamente diferentes (Tukey, $\mathrm{p}>0.05$ ) 

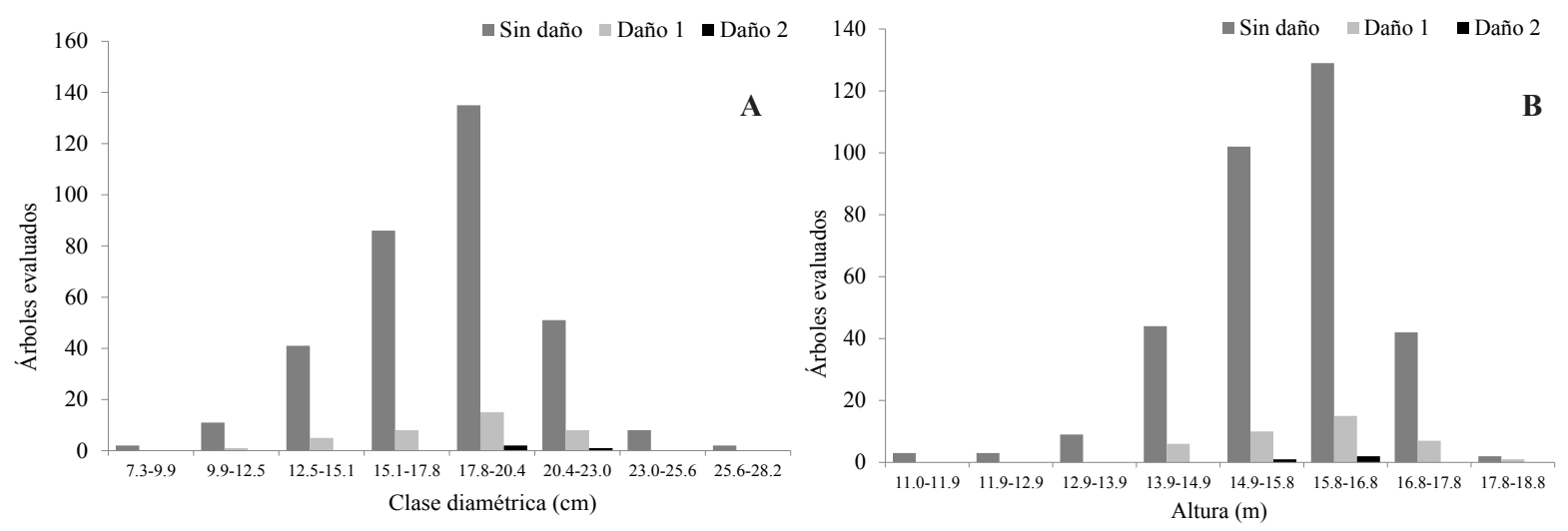

Figura 2. Incidencia de daño. A). Clase diamétrica y B). Altura. Buena Fe
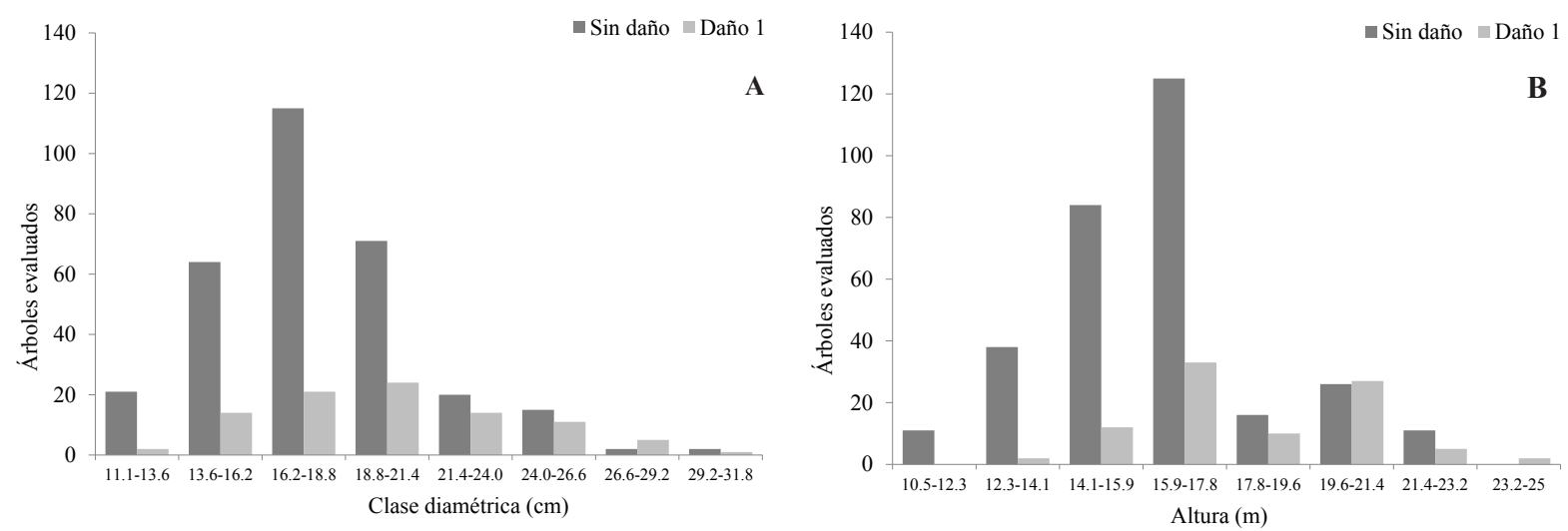

Figura 3. Incidencia de daño. A). Clase diamétrica, B). Altura. El Empalme

árboles muertos por C. ochromactonus se reporta (Stiwell et al., 2014). En El Empalme hubo más árboles por área evaluada, presentando una mayor densidad por hectárea. Este factor influye en la incidencia de daño o ataque de los escolítidos (Amman, 1973), lo que podría explicar en parte el porcentaje de incidencia. Solo en Buena Fe se registró la muerte regresiva de los árboles, esta corresponde a una zona intermedia, similar a lo reportado por Stilwell et al. (2014), con el mayor número de árboles muertos.

En Buena Fe, el Dap promedio de crecimiento fue 18 $\mathrm{cm}$, encontrándose desde 7.30 a $28.20 \mathrm{~cm}$, sin embargo, los daños se presentaron en árboles desde 11.20 a $21.70 \mathrm{~cm}$, con mayor incidencia de daño en árboles de $19.10 \mathrm{~cm}$ (17.80$0.40 \mathrm{~cm}$ ). La altura promedio fue de $15.70 \mathrm{~m}$, variando entre 11.50 a $18.30 \mathrm{~m}$, pero, el rango de daño se presentó desde 14.40 a $18.30 \mathrm{~m}$ con una incidencia mayor en árboles de $16.30 \mathrm{~m}$ (15.80-16.80 m). La mayor incidencia de daño se presentó en los árboles de crecimiento promedio en la plantación (Figura 2).

En El Empalme, el diámetro promedio fue de 18.60 $\mathrm{cm}$, con un rango de 11.00 a $31.80 \mathrm{~cm}$, pero, la preferencia de ataque fue en árboles con diámetro entre 12.30 a 30.50 $\mathrm{cm}$ con mayor incidencia de daño en árboles de 17.50 cm (16.20-18.80) y $20.10 \mathrm{~cm}(18.80-21.40)$. La altura promedio fue $16.70 \mathrm{~m}$, el rango fue entre 10.50 a $25.00 \mathrm{~m}$, pero los daños por C. ochromactonus van de 13.20 a $24 \mathrm{~m}$, con mayor incidencia en árboles de 16.80 m (15.90-17.80) y $20.50 \mathrm{~m}$ (19.60-21.40). La incidencia de ataque fue mayor en los árboles con crecimiento promedio (Figura 3). En las dos localidades, la incidencia de daño fue mayor en el rango de diámetros de mayor número de individuos, 17.80 a 20.30 $\mathrm{cm}$ para Buena Fe y 16.20 a $18.70 \mathrm{~cm}$ y 18.80 a $21.30 \mathrm{~cm}$ en El Empalme. Los diámetros obtenidos en las localidades son parecidos a los reportados por Stilwell et al. (2014) quienes indican que el diámetro promedio de árboles atacados o muertos fue de $22.10 \mathrm{~cm}$ en la plantación $a$, mientras, en la plantación $b$ fue de $16.90 \mathrm{~cm}$.

\section{Conclusiones}

Cochromactonus causa dos ataques iniciales, en el
fuste o ramas superiores de la balsa, y en este último,
dependiendo de la cantidad de perforaciones se producen
síntomas como marchitez y muerte del follaje, con un 
posterior ataque masivo en todo el fuste. Los ataques de $C$. ochromactonus están dirigido a árboles con diámetros de crecimiento promedio, 19.10 en Buena Fe y entre 17.50 y 20.10 en El Empalme.

\section{Agradecimientos}

$\mathrm{A}^{\mathrm{la}}$ la Secretaría de Educación Superior, Ciencia, Tecnología e Innovación (SENESCYT), beca 2011. A funcionarios de Escuela de Graduados de la Facultad de Ciencias Forestales y Recursos Naturales de la Universidad Austral de Chile, por permitir investigar una problemática de Ecuador. A la Universidad Técnica Estatal de Quevedo y a la empresa Plantabal S. A.- 3AComposites, quienes facilitaron la logística y actividades en campo para la evaluación.

\section{Bibliografía}

Amman, G. (1973). Population changes of the mountain pine beetle in relation to elevation. Environmental Entomology 2(4): 541-547.

Bonet, X., Coello, J. y Andrade, H. (2013). Generalidades de la madera de balsa como material para núcleos en la construcción de Sandwich PRFV. BALSEUROP ECUATO ESPAÑOLA, SL. Spain.

Brink, M. (2008). Ochroma pyramidale (cav. Ex Lam.) Urb. In: D. Louppe, A. A. Oteng-Amoako and M. Brink. (eds), PROTA (Plant Resources of Tropical Africa), Wageningen, Netherlands.

Castro, J. (2016). Aspectos biológicos y ecológicos de Coptoborus ochromactonus Smith y Cognato (Coleoptera: Scolytinae), y la relación de sus hongos asociados en la muerte regresiva de Ochroma pyramidale (Cav. Ex. Lam.) Urb. Tesis de Doctorado. Universidad Austral de Chile. Valdivia, Chile.

Fletcher, M. (1949). Balsa industry in Ecuador. Economic Geography 25(1): 47-54.

González, B., Molina, X., Navarrete, E., Sánchez, C. y Simba, L. (2010). Caracterización del cultivo de balsa (Ochroma pyramidale) en la provincia de Los Ríos Ecuador. Ciencia y Tecnología 3(2): 7-11.

Keneko, T., Tamaki, Y. and Takagi, K. (1965). Preliminary report on the biology of some Scolytid beetles, the tea root bores, Xyleborus germanus Blanford, attacking tea roots, and the tea stem bores Xyleborus compactus Eichhoff, attacking tea twigs. Japanese Journal of Applied Entomology and Zoology 9(1): 23-28.

Lee, J., Haack, R. and Choi, W. (2011). Attack pattern of Platypus koryoensis (Coleoptera: Curculionidae: Platypodinae) in relation to crown dieback of Mongolia oak in Korea. Environmental Entomology 40(6): 13631369.

Midgley, S., Blyth, M., Howcroft, N., Midgley, D. and Brown, A. (2010). Balsa: biology, production and econonomics in Papua New Guinea. ACIAR Technical Reports 73. Australian Centre for International Agricultural Research: Canberra, 98 pp.

Raffa, K., Phillips, T. and Salom, S. (1993). Strategies and mechanisms of host colonization by bark beetles. Beetle - pathogen interaction in conifer forest. In: Schowalter, T.; Filip, G. (eds). Beetle pathogenic interactions in conifer forest. Academic press limited. Cambridge, Great Britain. pp 103-120.

Reding, M., Oliver, J., Schultz, P. and Ranger, Ch. 2010. Monitoring flight activity of ambrosia beetle in ornamental nurseries with ethanol - baited traps: influence of trap height on captures. J. Environ. Hort 28(2):85-90.

Rudinsky, A. (1962). Ecology of Scolytidae. Annual Review of Entomology 7:327-348.

Stilwell, A., Smith, S., Cognato, A., Martinez, M. and Flowers, W. (2014). Coptoborus ochromactonus, n. sp. (Coleoptera: Curculionidae: Scolytinae), an emerging pest of cultivated balsa (Ochroma pyramidale) in Ecuador. Journal of Economic Entomology 107(2): 675-683

Weber, BC., and McPherson, J. 1983. Life history of the ambrosia beetle Xylosandrus germanus (Coleoptera: Scolytidae). Annals of the Entomological Society of America, 76(3): 455-462.

Wood, S. (2007). Bark and ambrosia beetles of South America (Coleoptera, Scolytidae). Center Brigham Young University. Provo, Utah. USA. 909 p.

Raffa, K., Phillips, T. and Salom, S. (1993). Strategies and mechanisms of host colonization by bark beetles. Beetle - pathogen interaction in conifer forest. In: Schowalter, T.; Filip, G. (eds). Beetle pathogenic interactions in conifer forest. Academic press limited. Cambridge, Great Britain. pp 103-120.

Rudinsky, A. (1962). Ecology of Scolytidae. Annual Review of Entomology 7:327-348.

Stilwell, A., Smith, S., Cognato, A., Martinez, M. and Flowers, W. (2014). Coptoborus ochromactonus, n. sp. (Coleoptera: Curculionidae: Scolytinae), an emerging pest of cultivated balsa (Ochroma pyramidale) in Ecuador. Journal of Economic Entomology 107(2): 675-683.

Wood, S. (2007). Bark and ambrosia beetles of South America (Coleoptera, Scolytidae). Center Brigham Young University. Provo, Utah. USA. 909 p. 